International Journal of Distributed and Parallel Systems (IJDPS) Vol.3, No.6, November 2012

\title{
QOS ENHANCED HYBRID MULTIPATH ROUTING PROTOCOL FOR MOBILE ADHOC NETWORKS
}

\author{
${ }^{1}$ P.Rama Devi and ${ }^{2}$ Dr.D.Srinivasa Rao \\ ${ }^{1}$ Associate Professor, Department of ECE, \\ Abhinav Hitech College of Engineering, Hyderabad. \\ pramadeviphdegmail.com \\ ${ }^{2}$ Professor \\ Department of ECE \\ JNTU CE, Hyderabad
}

\begin{abstract}
In Mobile ad hoc networks (MANET), the quality of service (QoS) depends on the available resources in the network and node mobility as mobility may result in frequent route failures. Some existing hybrid approach of multi-path routing technique rarely considers QoS metrics for path selection. In this paper, we propose a QoS enhanced hybrid multi-path routing protocol for MANET. In this protocol, topology discovery is performed proactively and route discovery is performed in the reactive manner. In proactive topology discovery phase, each node collects the battery power, queue length and residual bandwidth of every other nodes and stores in the topology information table (TIT). By exchanging the TIT among the nodes, the topology is discovered. When the source node wants to forward the data packet to the destination, it utilizes the reactive route discovery technique where the multiple paths are established using multi-path Dijkstra algorithm. When any intermediate node does not recognize the next 2-hop information from TIT towards destination, the new multi-path route discovery is performed. By simulation results, it is shown that the proposed approach reduces the overhead.
\end{abstract}

\section{KEY WORDS}

Mobile Adhoc Networks (MANET), Quality of service (QoS), Topology Information Table (TIT), Ad Hoc On-Demand Distance Vector (AODV)

\section{INTRODUCTION}

\subsection{Mobile Ad hoc Network (MANET)}

A self-determining system of mobile routers and related hosts link by the wireless routes is termed as mobile ad hoc networks (MANET). It does not contain any base stations. Alternatively, a function of routing is included in every mobile host and multi-hops possibly will be essential to permit one node to interact with another node over the ad hoc network owing to the restricted transmission range. There is a possibility that wireless topology of the network may get varied randomly and quickly as the routes travel arbitrarily and systemize themselves randomly. Hence, MANETs are illustrated as active, multi-hop and continuously modifying topology. [1]

Some examples of possible uses of a wireless ad-hoc network include military applications, law enforcement, emergency response efforts, commercial use, and education. [2]

\subsection{Multipath Vs Single Path Routing}

The process of transmitting the data packet from source to destination via wireless medium in mobile ad hoc networks is termed as routing. It becomes the major issue in ad hoc network, as it possess exclusive configuration.

DOI : $10.5121 /$ ijdps.2012.3608 
International Journal of Distributed and Parallel Systems (IJDPS) Vol.3, No.6, November 2012

\section{- Single Path Routing}

In case of single path routing, a single path is utilized to transmit the packets from the source to destination. The process of including the route information in the packet header corresponds to the dynamic source routing (DSR) protocol which is considered as source dependent single path routing algorithm. Whereas for ad hoc on-demand distance vector routing (AODV) protocol, the destination nodes information is included in the packet header and in order to transfer the data packets in single path, hop-by-hop packet forwarding mechanism is utilized. [3] Owing to the inconsistency of the wireless infrastructure and nodes mobility, single path routing protocols causes performance degradation in mobile networks.

\section{- Multipath Routing}

The process of discovering multiple routes among the distinct source and single destination at the time of single route discovery corresponds to multi-path routing. [4] In MANET, the prevailing issues such as scalability, security, network lifetime, etc can be handled by the multipath routing protocols. [5] This protocol enhances the end-to-end throughput and offers load balancing in MANETs.

\subsection{Quality of Service (QoS)}

The network offering a group of service necessities to some traffic for fulfilling the users needs related to that traffic is termed as Quality of service (QoS). [6] The main idea of QoS is to assure certain pre-defined service performance limitations of the user with respect to end-to-end delay, available bandwidth, packet loss probability etc. [7]

\subsubsection{Need for QoS Routing}

- The routing in MANET turns out to be more difficult work owing to recurrent modification in network topology and network resource deficiency together in wireless medium as well as mobile nodes. Consequently, routing will lead to route breakage frequently. Thus for the routing protocol maintaining QoS for ad hoc networks, link failure should also be taken into account for enhancing the system performance. [8]

- QoS routing protocols should fulfill end-to-end QoS needs with respect to bandwidth or delay along with the route discovery from source to destination. [9] The major function of QoS routing protocol is to calculate the paths appropriate for various category of traffic created by different applications and also it maximizes the network resources usage.

\subsubsection{QoS Metrics}

This metrics is required to measure the quality of service. The appropriate metrics are as follows [10]

- Minimum average throughput (bps)

The total number of data rates distributed to all network terminals is termed as aggregate throughput.

- Maximum packet delay bound (s)

The collection of queuing and MAC delays at each node along with propagation delay which is comparatively short is called as packet delay. 
International Journal of Distributed and Parallel Systems (IJDPS) Vol.3, No.6, November 2012

- Maximum delay jitter bound

The difference between the upper bound on delay and total minimum delay found using the collective propagation and duration of packet transmission is termed as delay jitter. Alternatively it can be defined as difference of the absolute packet delay.

- Maximum packet loss ratio (PLR) bound

It represents the most tolerable fraction of the generated data packets that are lost en-route. The packet loss occurs during the following situations

- Overflow of buffer during congestion

- Rise of re-transmission limit at the time of poor channel quality

- Time-out condition during waiting for discovery of new route.

\subsection{QoS in MANETs}

Quality of service in MANET relies on both existing resources in the network and mobility rate of such resources. These metrics are considered since the mobility may cause route failure and MANET holds only limited resources when compared to the fixed networks. Hence excess metrics need to be considered to confine with quality of the links among nodes. This quality ought to be a function of resource availability existing in wireless and mobile environment. [8] Also QoS-based routing metric for MANETs must include minimum available bandwidth and end-to-end latency together with congestion around a link. [11]

\subsection{Issues of QoS and Multipath Routing Protocol}

\subsubsection{Quality of Service (QoS) Issues}

- It is difficult to guarantee QoS to MANET applications owing to the inconsistent wireless channel, deficiency in centralized control, channel access contention, mobility of node and admission control. [10]

- In MANET, the nodes don't possess any limitation towards mobility. There is a recurrent and random variation in the network topology. The QoS admission might experience serious issues owing to recurrent path failure and partitions of the network. [6]

- The mobile nodes in MANET possess inadequate power and they are liable to repeated node failures. It results in variations of network topology, network partitions, packet losses and minimum signal quality. [6]

- The main necessity of QoS is link quality of the network. However a minimum capacity resource that differs based on time can cause maintenance of routing information more complex.

\subsubsection{Multipath Routing Issues}

Multipath routing has some disadvantages: [5]

\section{- Route request storm}

A huge quantity of route request messages are created by the multipath reactive routing protocols. When the intermediate nodes requires to process the duplicate request messages, there is a chance of unnecessary overhead packets be set up in the networks. 
International Journal of Distributed and Parallel Systems (IJDPS) Vol.3, No.6, November 2012

\section{- Inefficient route discovery}

Certain multipath routing protocols avoid intermediate node from forwarding a reply from its route cache in order to determine node-disjoint or link disjoint paths. Hence the source has to wait till it gets a reply from destination. Thus the process of route discovery performed by the multipath routing protocol needs more time when compared with DSR or AODV protocols.

\subsection{Problem Identification}

There exist protocols for QoS aware routing in literature but what kind of link metric to use and how it can be used properly in MANET are still open issues. Also Multipath routing based on hybrid approach (proactive and reactive) rarely consider QoS metrics for path selection. In [5], the Multipath optimized link state routing protocol uses OLSR as the base routing protocol and uses both proactive and reactive approaches for route discovery. But it does not consider the QoS based link quality metrics like power, bandwidth, delay etc. in routing table formation.

In order to avoid these issues, we propose a QoS enhanced hybrid multi-path routing protocol which considers link quality as a QoS metric.

\section{RELATED WORKS}

Luo Liu et al. [7] have proposed architecture for assuring QoS based on Node-Disjoint multipath routing protocol (NDMR) in mobile ad hoc networks. The problem related to provisioning of QoS is extremely difficult task in MANETs. But the multiple node-disjoint paths help in assigning the packets to paths in a best possible method to handle some limitations. The proposed methodology offered limitations and also compared the functioning in variation circumstance of NDMR. This method also determined ways of establishing NDMR with the help of queue length field and updates route packets for permitting QoS computations over node-disjoint paths.

Chunxue Wu et al. [9] have proposed Q-AOMDV protocol for ad hoc networks. The proposed protocol with path preference probability calculates the delay, bandwidth, hop count for choosing the path for forwarding the packet. The provision of multiple paths is more efficient in ad hoc networks since the source can just utilize the existing routes in case of any route failure instead of carrying out route recovery process.

Fujian Qin et al. [12] have proposed a new Multipath source routing protocol with bandwidth and reliability constraints for MANET. In order to get the multipath routing, they expand DSR's routing discovery and maintenance technique. To attain a better cooperation among load balancing and network overhead, an ultimate count of multipath route is examined. Also, per packet granularity is utilized to allocate the packets from multiple links between the paths in MSR.

Sanguankotchakorn et al. [13] have proposed NQoS AODV by altering the conventional AODV. NQoS AODV upholds a routing table which frequently offers routes thus minimizing the average delay. This approach increases the packet delivery ratio since it upholds the QoS information and observes for the path fulfilling QoS necessities of the applications. Further it forwards a smaller number of control packets to maintain route discovery and route failure which causes reduced control overhead.

Nityananda Sarma et al. [14] have proposed a Route Stability based QoS Routing (RSQR) protocol in Mobile Ad Hoc Networks (MANETs) which is an extension of QoS routing with throughput and delay constraints. In order to guarantee the suitable data path for adequate longer duration in MANET, they have proposed easy model for measuring the link stability and route stability depending on received signal strengths. Some additional fields in route request/ reply 
International Journal of Distributed and Parallel Systems (IJDPS) Vol.3, No.6, November 2012

packets is taken into consideration so that the route stability information can be used to choose a route with increased stability when compared to all possible routes among existing source destination pair.

Kun-Ming Yu et al. [15] have proposed a new protocol (ARMBR) to enhance the prevailing on demand routing protocols. This is performed by building multiple backup routes. During the modulation in network topology, the protocol can transfer the data packets actively via backup routes. In addition, they have developed an analytical model to determine the reconnection probability of the proposed algorithm.

Samuel Pierre et al. [16] have proposed a new approach based on a mobile routing backbone for supporting Quality of service (QoS) in MANETs. This proposed protocol allocates the traffic inside the network as per the existing network traffic level and nodes processing loads. The QoS support is recognized with the help of communicating packets possessing particular necessities to nodes that are loaded with more resources and connected through stable links.

\section{Hybrid QoS Aware Multipath Routing Protocol}

\subsection{Overview}

In this paper, we propose a hybrid QoS aware multi-path routing protocol for MANET. In this technique, topology discovery is performed proactively and route discovery is performed in the reactive manner. In topology discovery phase, each node learns the battery power, queue length and residual bandwidth of every other nodes and stores in the topology information table (TIT). By exchanging the TIT among the nodes, the topology is discovered. When source wants to forward a data packet to destination, it verifies TIT and computes the link metric (LM) using the data in its TIT. The source chooses the nodes with minimum LM and initiates the packet transfer through the chosen node within 2-hop. The Multi-path Dijkstra algorithm is employed to transmit the data through multiple paths with the nodes holding minimum link metric. When any intermediate node does not recognize the next 2-hop information from TIT towards destination, then it propagates route request (RREQ) message to all the nodes as per any reactive multi-path routing protocol like AOMDV. Then route reply (RREP) messages are sent along the reverse routes to the source, using which it can setup the best path to the destination. Whenever the new path is discovered reactively, the source then updates its TIT.

\subsection{Estimation of Route Metrics}

\subsubsection{Estimation of Residual Battery power}

After time $t$, the power consumed by the node $(P(t))$ is computed as follows.

$$
P(t)=D P_{t x} * \lambda+D P_{r e} * \eta
$$

Where $\mathrm{DP}_{\mathrm{tx}}=$ Number of data packets transmitted by the node after time $t$

$\mathrm{DP}_{\mathrm{re}}=$ Number of data packets received by the node after time $t$

$\lambda$ and $\eta$ are constants in the range of $[0,1]$.

If $P_{i}$ denotes the initial power of a node, the residual power $P_{R}$ of a node at time $t$, can be calculated as:

$$
P_{R}=P_{i}-P(t)
$$

\subsubsection{Estimation of Queue length}

The traffic load of the mobile node can be demonstrated by knowing the number of packets in the queue [17]. When excess traffic flows through the mobile nodes, it reveals that the interface 
queue possesses more packets. Thus average queue size that specifies the traffic load of the node can be computed as follows.

$$
Q L=\delta * Q L_{0}+(1-\delta) * Q L_{c}
$$

where $Q L=$ Average queue length

$Q L_{c}=$ Current queue length,

$Q L_{0}=$ Old queue length

$\delta=$ Constant in the range $[0,1]$

\subsubsection{Estimation of Residual Bandwidth}

Every node within the interference range holds the sufficient bandwidth for transmitting the data without congestion. Thus it is necessary to familiarize with the local and neighboring nodes within the interference range. Any node that has necessity to transmit the data must consider local bandwidth and interference range mutually. The process of predicting the bandwidth of local and neighboring nodes is explained below.

Since the bandwidth is shared among neighboring nodes, by taking channel into consideration, the nodes calculates bandwidth based on the ratio of idle and busy times projected for predefined interval of time $(t)$

The local bandwidth $\left(B_{1}\right)$ is estimated as follows.

$$
B_{1}=C_{c h} *\left(\frac{t_{i}}{t}\right)
$$

Where $C_{c h}=$ channel capacity and

$$
t_{i}=\text { idle time in } t .
$$

As the information regarding the neighboring nodes is gathered previously, the minimum bandwidth $\left(B_{\min }\right)$ of all nodes within the transmission range can be recognized. Thus the residual bandwidth $\left(B_{R}\right)$ is defined as the difference between $\left(B_{\min }\right)$ and $B_{1}$ and is stored in the residual bandwidth register.

\subsection{Multi-path Routing}

The main goal of this multipath algorithm is to construct a group of $\mathrm{N}$ routes devoid of loops, connecting source (S) and destination (D).

In the source node, the multipath optimized link state routing protocol holds a updated flag $Z_{i}$ for every possible node in the network for recognizing the validity of the routes related to the node. Primarily, $Z_{i}$ is assigned to be false which reveals that either there is no route related to the destination or renewal process is required. The condition to obtain the multiple paths for any node $n_{i}$ is as follows.

If $Z_{i}=$ false,

Then

The node executes multipath Dijkstra algorithm to obtain the multiple paths to $n_{i}$, store it in the multipath routing table, and performs the renewal of corresponding $Z_{i}$ to be true. 
Else

The node will discover a valid route to $n_{i}$ in the multipath routing table.

End if

\section{Multipath Dijkstra Algorithm}

Let ST represent the source tree.

Let $\mathrm{w}_{\mathrm{r}}$ represent the opposite edge of arc $\mathrm{w}$.

Let $h(w)$ offers the vertex edge to $w$ points.

$\mathrm{F}$ (ST, D) is the function that obtains the shortest path to D from ST.

$\mathrm{F}_{\mathrm{p}}$ is used to increase the costs of $\mathrm{w}$ that belong to the previous path $\left(P_{i}\right)$

$\mathrm{F}_{\mathrm{w}}$ is used to increase the costs of $\mathrm{w}$ that lead to vertices of $P_{i}$.

The algorithm is applied to a graph $G=\{\psi, \tau, \sigma\}$ to compute N routes in $\mathrm{G}$ from S to D.

Where $\psi=$ set of vertices

$$
\begin{aligned}
& \tau=\psi * \psi=\text { set of arcs } \\
& \sigma: \psi \rightarrow K^{+}=\text {strictly positive cost function. }
\end{aligned}
$$

$\sigma_{1}=\sigma$

$G_{1}=G$

For $i \leftarrow 1$ to $N$

do

$$
S T_{i} \leftarrow \operatorname{Dijkstra}\left(G_{1}, S\right)
$$

$P_{i} \leftarrow F\left(S T_{i}, D\right)$

For all arcs $\mathrm{w}$ in $\tau$

If $\mathrm{w}$ is in $P_{i}$ or Reverse (w) is in $P_{i}$

Then

$$
\sigma_{i+1}(w) \leftarrow F_{p}\left(\sigma_{i}(w)\right)
$$

Else if the $\mathrm{h}(\mathrm{w})$ is in $P_{i}$

Then

$$
\sigma_{i+1}(w) \leftarrow F_{w}\left(\sigma_{i}(w)\right)
$$

Else

$$
\sigma_{i+1}(w) \leftarrow \sigma_{i}(w)
$$

End if

End for

$G_{i+1} \leftarrow\left(\psi, \tau, \sigma_{i+1}\right\}$

End for

$\operatorname{Return}\left(P_{1}, P_{2}, P_{3}, \ldots, P_{N}\right)$

\subsection{Hybrid QoS Aware Multipath Routing Protocol}

The proposed protocol is a hybrid protocol that combines the features of both proactive and reactive protocol. It involves two phases. 
Phase 1: Proactive topology discovery

Phase 2: Reactive Route Discovery Phase.

\subsubsection{Phase 1 Proactive topology discovery}

\section{Step 1}

Each node deployed in the network periodically exchanges a topology message with its neighbor nodes.

\section{Step 2}

By exchanging the topology messages, every node measures QoS metrics such as the residual battery power $\left(\mathrm{P}_{\mathrm{R}}\right)$, queue length $(\mathrm{QL})$ and residual bandwidth $\left(\mathrm{B}_{\mathrm{R}}\right)$ of its neighbor nodes. (Explained in section 3.1.1-3.1.3)

\section{Step 3}

After the measurement of QoS metrics, each node gathers information about other nodes and stores in a topology information table (TIT). Thus TIT holds the source node ID, 1-hop and 2hop neighbor node ID, residual battery power $\left(\mathrm{P}_{\mathrm{R}}\right)$, queue length $(\mathrm{QL})$ and residual bandwidth $\left(\mathrm{B}_{\mathrm{R}}\right)$ of each node along with the 2-hop neighborhood information.

Table 1. Topology Information Table (TIT)

\begin{tabular}{|c|c|c|c|c|c|}
\hline $\begin{array}{c}\text { Source } \\
\text { Node ID }\end{array}$ & $\begin{array}{c}\text { 1-hop } \\
\text { neighbor } \\
\text { node ID }\end{array}$ & $\begin{array}{c}\text { 2-hop } \\
\text { neighbor } \\
\text { node ID }\end{array}$ & $\begin{array}{c}\text { Residual } \\
\text { Energy }\end{array}$ & $\begin{array}{c}\text { Queue } \\
\text { length }\end{array}$ & $\begin{array}{c}\text { Residual } \\
\text { bandwidth }\end{array}$ \\
\hline
\end{tabular}

\section{Step 4}

The TIT value is exchanged among the nodes, and utilizing the updated node information, the topology is discovered.

\subsubsection{Phase 2: Route Discovery Phase}

When the source $\mathrm{S}$ has a necessity to establish the route towards $\mathrm{D}$ through the intermediate nodes, route discovery phase is executed. The steps involved in the route discovery are as follows.

\section{Step 1}

When $\mathrm{S}$ requires forwarding a data packet to $\mathrm{D}$, initially it verifies the TIT.

\section{Step 2}

After verification, $\mathrm{S}$ gathers all the information about the nodes towards D.

Step 3

$\mathrm{S}$ computes the link metric (LM) using the data in its TIT which is as follows

$$
L M=\frac{\eta * Q L}{\left(\alpha * P_{R}\right)+\left(\beta * B_{R}\right)}
$$

Where $\alpha, \beta$ and $\eta$ represents the normalization factor.

\section{Step 4}

$\mathrm{S}$ chooses the nodes with minimum LM and initiates the packet transfer through the chosen node within 2-hop. The Multipath Dijkstra algorithm (Explained in section 3.1.4) is employed to 
transmit the data through multiple paths with the nodes holding minimum link metric. The process is demonstrated using Figure 1

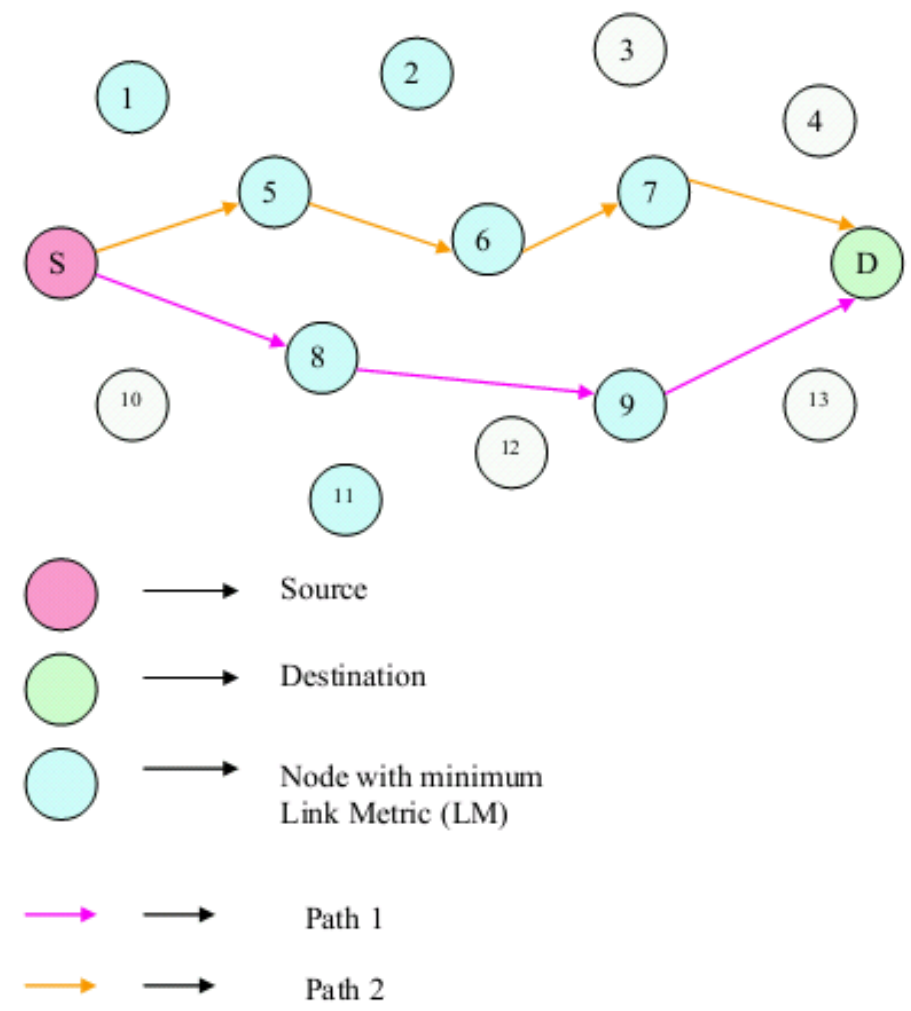

Figure 1. Multipath establishment using Multipath Dijkstra Algorithm

Figure 1 represents the establishment of multiple paths using Multipath Dijkstra Algorithm. The source after computing the LM, finds that the nodes $1,2,5,6,7,8$, and 9 possess minimum LM. Then utilizing the multi-path Dijkstra algorithm, the source forwards the data packet towards the destination. The path 1 is chosen as the primary path since the destination is reached within its two hop neighbors. The path 2 is chosen as the backup path.

\subsection{Reactive Multi-path Routing}

When any intermediate node $\left(\mathrm{n}_{\mathrm{i}}\right)$ does not recognize the next 2-hop information from TIT towards destination, the reactive multi-path routing protocol (like AOMDV) is performed for route discovery. The steps involved are as follows.

\section{Step 1}

The intermediate node $\left(n_{i}\right)$ broadcasts route request (RREQ) message to all neighboring nodes through the eligible links towards the destination (D) and waits for the route reply (RREP) message.

\section{Step 2}

$$
n_{i} \stackrel{R R E Q}{\longrightarrow} n_{\text {neigh }}
$$

When any $n_{\text {neigh }}$ possessing an eligible route receives the RREQ, it replies requested $n_{i}$ with the RREP message. 
International Journal of Distributed and Parallel Systems (IJDPS) Vol.3, No.6, November 2012

\section{Step 3}

$$
n_{i} \stackrel{R R E P}{\longleftarrow} n_{n e i g h}
$$

On receiving the RREP, $\mathrm{n}_{\mathrm{i}}$ computes its link metrics and compares the link metrics with the value already stored in its TIT, and if satisfies the requirement, it start sending data following that route and discard duplicate RREP packets received in other feasible paths.
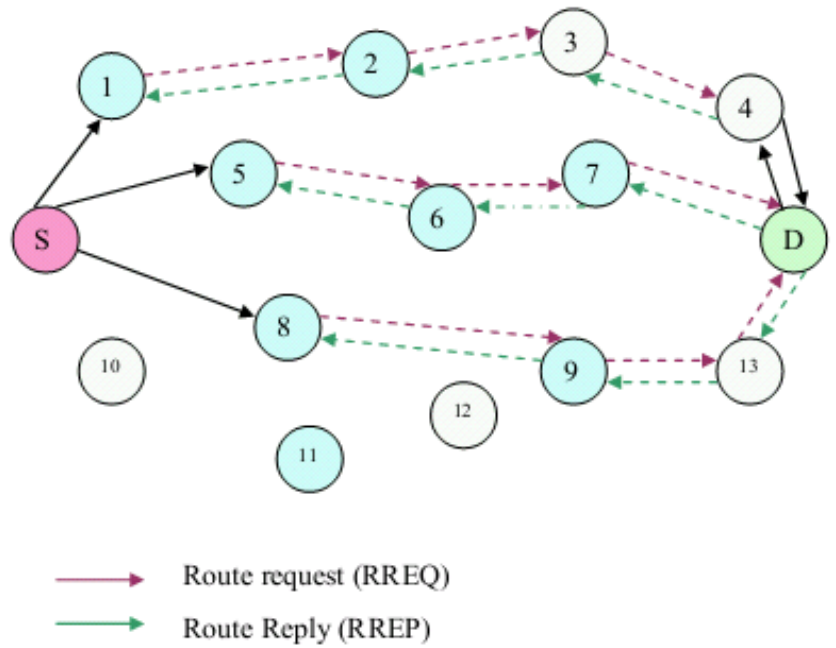

Figure 2. Route request and route reply phase

From Figure 2, the intermediate node 5, 1, 8 starts broadcasting RREQ packets. Upon receiving the RREQ packet, the neighbor nodes reply with the RREP packet to the requested node. The node 5, 1 and 8 verifies the RREP and computes its link metrics and if the link metrics matches with the value already stored in its TIT, it start sending data following that route and discard other duplicate RREP packets.

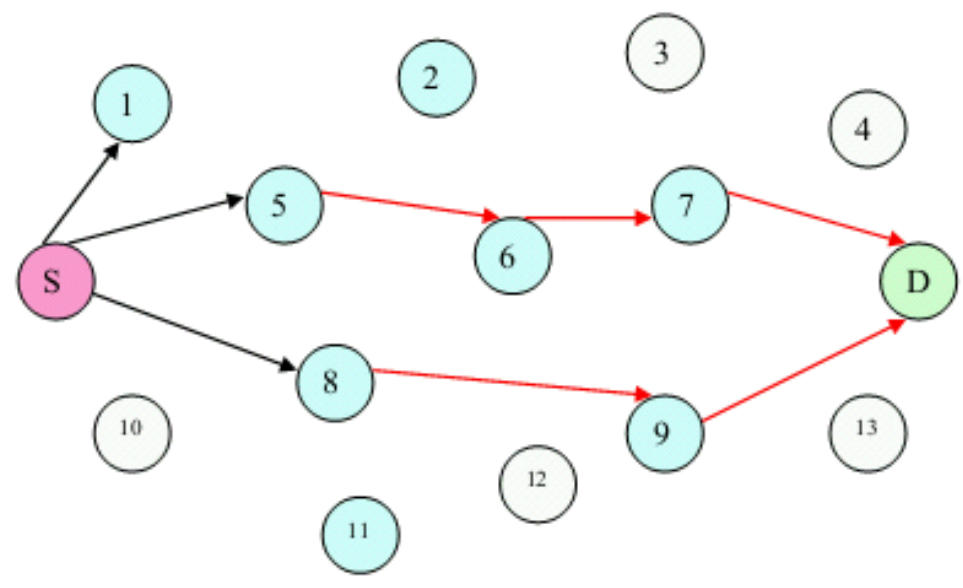

$\longrightarrow \quad$ Available multiple paths

Figure 3. Reactive Multi-path Routing 
International Journal of Distributed and Parallel Systems (IJDPS) Vol.3, No.6, November 2012

\subsection{Route Recovery}

The mobility, congestion, packet collisions, node failures etc can result in link failures in ad hoc networks.

When any node detects a link failure, it broadcasts the route error (RERR) packets to its neighbor nodes.

$$
n_{i} \stackrel{R R E R}{\longrightarrow} n_{\text {neigh }}
$$

Link failure

The neighbor nodes re-broadcast the packets until the source nodes receive the RRER packets.

When a source node receives the RRER, it eliminates all entry in its routing table that utilizes the broken link and uses the shortest backup paths as primary paths. The RRER packets should contain the information about the primary path failure as well as the backup path failure.

In case all the backup paths are broken, the source node will initiate a route discovery process (Explained in section 3.2.2).

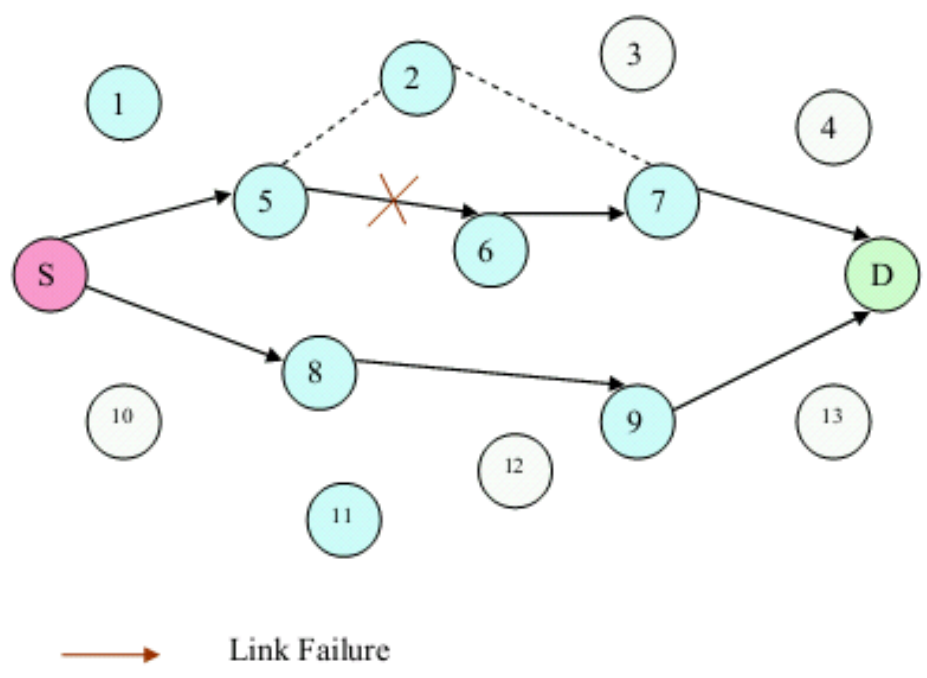

Figure 4. Route Recovery

From Figure 4, it is shown that there is link failure between the nodes 5 and 6 . Hence the node 5 broadcast the RERR packet to all the nodes. When $\mathrm{S}$ receives the RERR packet, it eliminates that route and uses the shortest backup paths i.e. through the node 2 as primary path.

\section{Simulation Results}

\subsection{Simulation Parameters}

We use NS2 [19] to simulate our proposed QoS enhanced hybrid multi-path routing protocol (QEHMR) protocol. In this simulation, the channel capacity of mobile hosts is set to the value of 2 Mbps. We use the distributed coordination function (DCF) of IEEE 802.11 for wireless LANs as the MAC layer protocol. It has the functionality to notify the network layer about link breakage. In our simulation, the number of nodes is varied as 30, 50, 70, 90 and 110. The mobile nodes move in a 1250 meter x 1250 meter square region for 50 seconds simulation time. 
International Journal of Distributed and Parallel Systems (IJDPS) Vol.3, No.6, November 2012

We assume each node moves independently with the same average speed. All nodes have the same transmission range of 250 meters. In our simulation, the speed is varied from $10 \mathrm{~m} / \mathrm{s}$ to $40 \mathrm{~m} / \mathrm{s}$. Random Way Point mobility model is used. The simulated traffic is Constant Bit Rate (CBR).

Our simulation settings and parameters are summarized in table 1.

Table 1. simulation parameters

\begin{tabular}{|c|c|}
\hline No. of Nodes & $30,50,70,90 \& 110$ \\
\hline Area & $1250 \times 1250$ \\
\hline MAC & 802.11 \\
\hline Radio Range & $250 \mathrm{~m}$ \\
\hline Simulation Time & $50 \mathrm{sec}$ \\
\hline Traffic Source & $\mathrm{CBR}$ \\
\hline Rate & $250 \mathrm{~kb} / \mathrm{s}$ \\
\hline Packet Size & $512 \mathrm{~B}$ \\
\hline No. of connections & 7 \\
\hline Mobility Model & Random Way Point \\
\hline Speed & $10,20,30 \& 40 \mathrm{~m} / \mathrm{s}$ \\
\hline Pause time & $5 \mathrm{~seconds}$ \\
\hline RxPower & 0.395 \\
\hline TxPower & 0.660 \\
\hline IdlePower & 0.035 \\
\hline Initial Energy & 10.3 \\
\hline
\end{tabular}

\subsection{Performance Metrics}

We evaluate performance of the new protocol mainly according to the following parameters. We compare the MPOLSR [5] routing protocol with our proposed QEHMR protocol.

Average Packet Delivery Ratio: It is the ratio of the number of packets received successfully and the total number of packets transmitted.

Average end-to-end delay: The end-to-end-delay is averaged over all surviving data packets from the sources to the destinations.

Control overhead: The control overhead is defined as the total number of routing control packets normalized by the total number of received data packets.

Throughput: It is the number of packets successfully received by the receiver.

Energy Consumption: It is the total amount of energy consumed by the nodes during the data transmission.

The simulation results are presented in the next section.

\subsection{Results \& Analysis}

A. Effect of varying Number of Nodes

Initially we vary the number of nodes as 30, 50, 70, 90 and 110 . 
International Journal of Distributed and Parallel Systems (IJDPS) Vol.3, No.6, November 2012

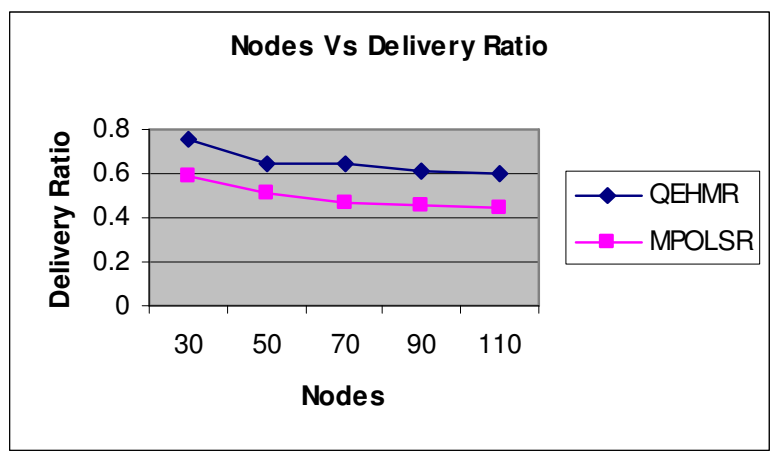

Figure 5. Nodes Vs Delivery Ratio

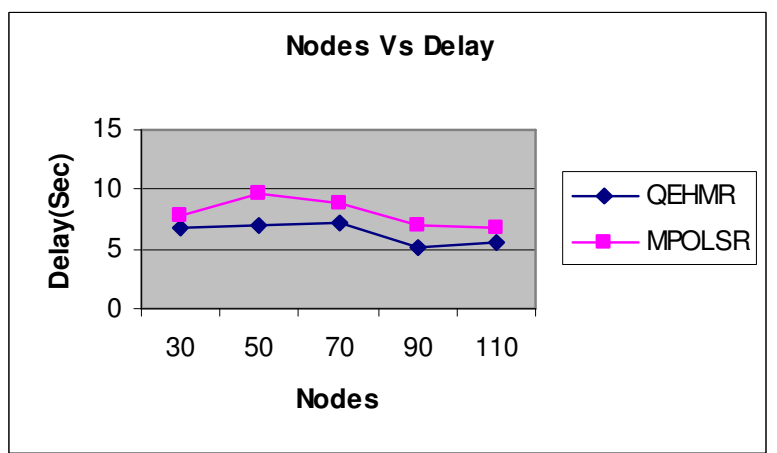

Figure 6. Nodes Vs Delay

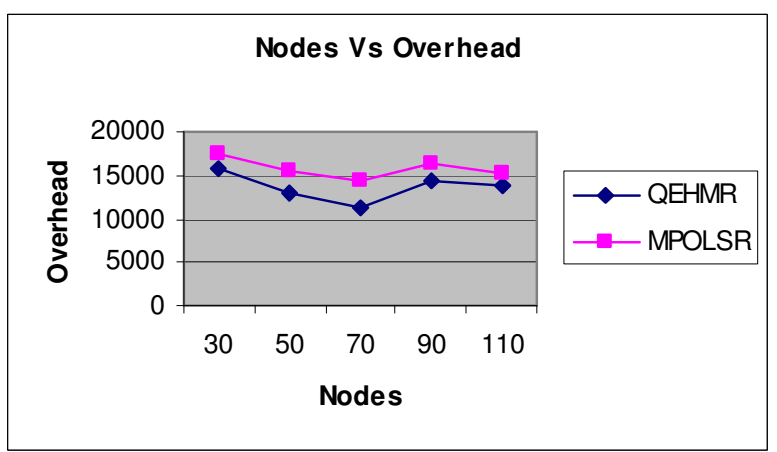

Figure 7. Nodes Vs Overhead 
International Journal of Distributed and Parallel Systems (IJDPS) Vol.3, No.6, November 2012

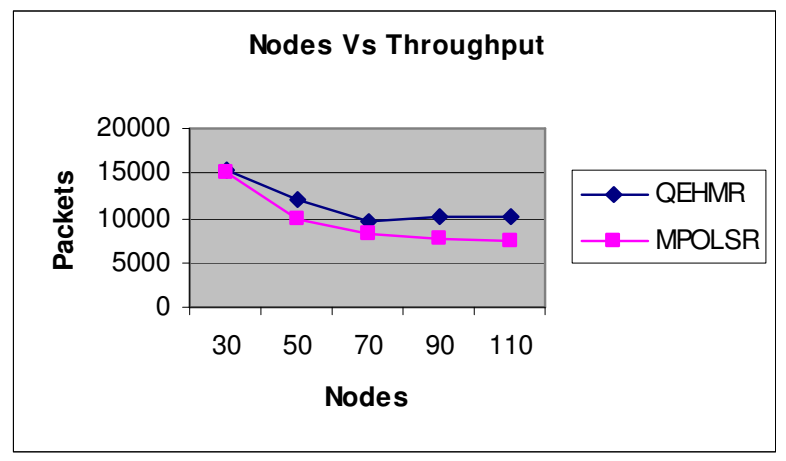

Figure 8. Nodes Vs Throughput

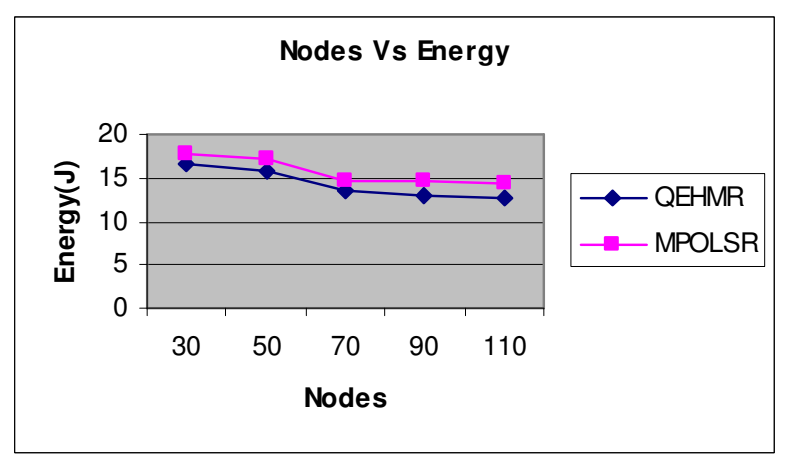

Figure 9. Nodes Vs Energy

When the number of nodes is increased from 30 to 110, the throughput and packet delivery ratio begin to reduce, as there is chances of more collisions.

Figure 5 and 8 show the results of average packet delivery ratio and throughput, respectively for the increased the nodes scenario. Clearly our QEHMR protocol achieves $23 \%$ better packet delivery ratio and $17 \%$ throughput than the POLSR since the proactive routing is done based on the QoS parameters bandwidth and queue length.

Figure 6 shows the results of average end-to-end delay for the increasing number of nodes. The figure depicts that delay increases when the nodes are increased from 30 to 70 , and then it reduces beyond 70 nodes. This is due to fact that the proactive routing couldn't' discover more shortest paths, since the nodes are sparse. From the results, we can see that QEHMR protocol has of $21 \%$ lower delay than the MPOLSR protocol.

Figure 7 shows the results of routing overhead versus number of nodes. The routing overhead decreases up to 70 nodes and increases beyond that since after 70 nodes, reactive routing is applied, rather than proactive.

From the results, we can see that QEHMR protocol produces 13\% less routing overhead than the MPOLSR protocol, since QEHMR uses the hybrid approach for route discovery.

Figure 9 show the results of energy consumption for the varying nodes scenario. Since battery power in considered as one of the QoS parameters while constructing the topology information table, the energy consumption for QEHMR protocol is 9\%less than MPOLSR. 
International Journal of Distributed and Parallel Systems (IJDPS) Vol.3, No.6, November 2012

\section{B. Effect of varying the Speed}

In our second experiment we vary the node speed as $10,20,30,40 \mathrm{~m} / \mathrm{s}$.

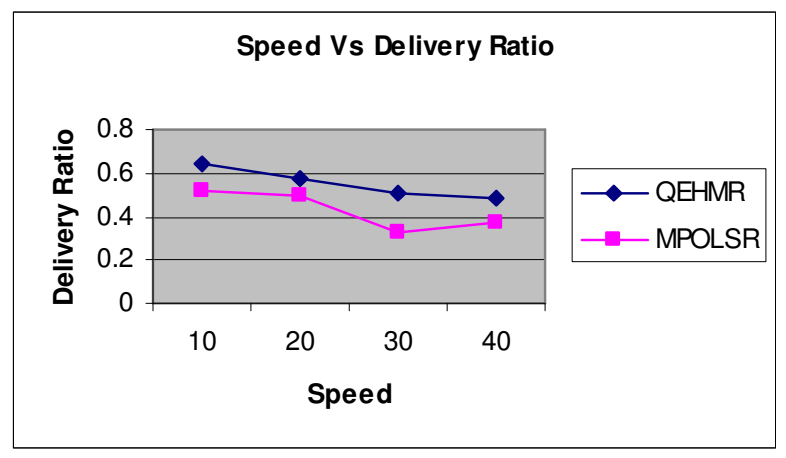

Figure10. Speed Vs Delivery Ratio

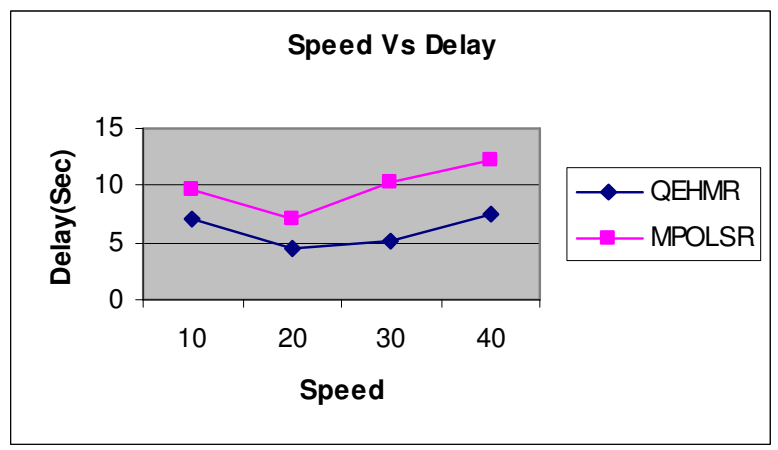

Figure11. Speed Vs Delay

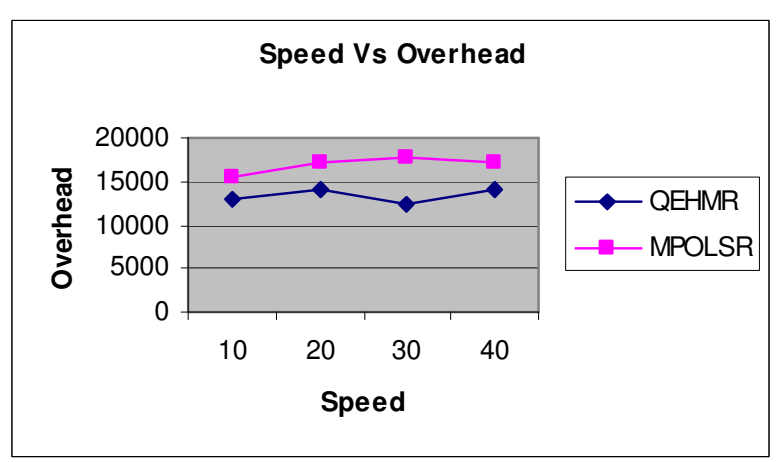

Figure12. Speed Vs Overhead 
International Journal of Distributed and Parallel Systems (IJDPS) Vol.3, No.6, November 2012

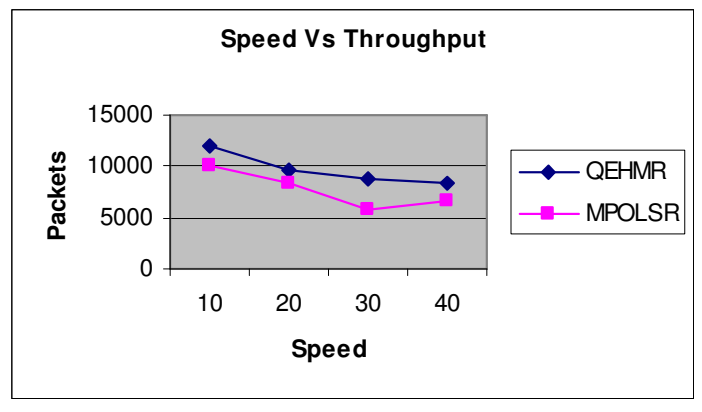

Figure13. Speed Vs Throughput

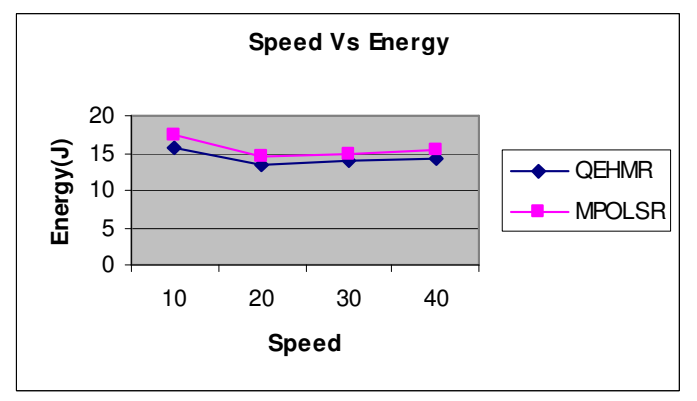

Figure 14. Speed Vs Energy

Increase in speed of the nodes results in more disconnections and hence the throughput and packet delivery ratio decreases. Figure 10 and 13 show the results of average packet delivery ratio and throughput, respectively for the varying speed scenario. Clearly our QEHMR protocol achieves better $22 \%$ packet delivery ratio and $20 \%$ throughput than MPOLSR, since it is having the route recovery mechanism.

Because of the route recovery procedures, the delay begins to increase when the speed increase. Figure 11 shows the results of average end-to-end delay for the increasing the node speed. From the results, we can see that QEHMR protocol has 38\% lower delay than the MPOLSR protocol.

Figure 12 shows the results of routing overhead versus speed. We can see that the overhead is increasing when the speed is increased from 10 to $40 \mathrm{~m} / \mathrm{s}$. It shows that QEHMR protocol produces $20 \%$ less routing overhead than the MPOLSR protocol, because of its hybrid nature.

Figure 14 show the results of energy consumption for the varying speed scenario. Clearly our QEHMR protocol has 7\% less energy consumption than MPOLSR.

\section{CONCLUSION}

In this paper, we have proposed a QoS enhanced hybrid multi-path routing protocol for MANET. In this technique, topology discovery is performed proactively and route discovery is performed in the reactive manner. In topology discovery phase, each node learns the battery power, queue length and residual bandwidth of every other nodes and stores in the topology information table (TIT). By exchanging the TIT among the nodes, the topology is discovered. When source wants to forward a data packet to destination, it verifies TIT and computes the link metric (LM) using the data in its TIT. The source chooses the nodes with minimum LM and initiates the packet transfer through the chosen node within 2-hop. The Multipath Dijkstra algorithm is employed to transmit the data through multiple paths with the nodes holding 
International Journal of Distributed and Parallel Systems (IJDPS) Vol.3, No.6, November 2012

minimum link metric. When any intermediate node does not recognize the next 2-hop information from TIT towards destination, the reactive multi-path routing protocol is performed for route discovery. By simulation results, it is shown that the proposed approach reduces the overhead.

\section{REFERENCES}

[1] Premalatha.J and Bala Subramanie.P, "Enhancing the quality of service in MANETs by effective routing", ICWCSC, 2010.

[2] Moon Jeong Kim, Dong Hoon Lee, and Young Ik Eom, "Enhanced Non-disjoint Multi-path Source Routing Protocol for Wireless Ad-Hoc Networks", Proceedings: International conference on Computational science and its applications - Volume Part III (ICCSA), 2007.

[3] Melike Oz Pasaogullari, Catherine M. Harmonosky and Sanjay Joshi, "Node Independent Multipath Routing Algorithm for MANET'.

[4] Chengyong Liu, Kezhong Liu, Layuan Li, "Research of QoS -aware Routing Protocol with Load Balancing for Mobile Adhoc Networks", WiCom' 08, 4th International Conference on Wireless communication, 2008.

[5] Yi, J., Adnane, A., David, S. and Parrein, B.," Multipath optimized link state routing for mobile ad hoc networks", Ad Hoc Networks 9, pp-28-47, 2011.

[6] Satyanarayana, D. and Rao, S.V., "Link Failure Prediction QoS Routing Protocol for MANET", Information and Communication Technology in Electrical Sciences, 2007.

[7] Luo Liu, Cuthbert, L.,"A Novel QoS in Node-Disjoint Routing for Ad-Hoc Networks", IEEE ICC Workshop, 2008.

[8] Navid Nikaein and Christian Bonnet, "A Glance at Quality of Service Models for Mobile Ad-Hoc Networks", DNAC, 2002.

[9] Chunxue Wu, Fengna Zhang and Hongming Yang, "A Novel QoS Multipath Routing in MANETs", International Journal of Digital Content Technology and its Applications, 2010.

[10] Lajos Hanzo II. And Rahim Tafazolli, “Admission Control schemes for 802.11-Based Multi-hop Mobile Adhoc Networks: A Survey”, IEEE Communications and Surveys, 2009.

[11] S.Venkatasubramanian and Dr.N.P.Gopalan, "A QoS Based Robust Multipath Routing Protocol for Mobile Ad-hoc Networks", International Journal of Engineering and Technology, 2009.

[12]Fujian Qin and Youyuan Liu, "Multipath Based QoS Routing in MANET", Journal Of Networks, 2009.

[13] Amina Akthar and Teerapat Sanguankotchakorn," Modified AODV for Multi-Constrained QoS Routing and Performance Optimization in MANET”, IEEE ECTI-CON, 2010.

[14] Nityananda Sarma and Sukumar Nandi, "Route Stability Based QoS Routing in Mobile Adhoc Networks”, Wireless Personal Communication, Vol-54, pp-203-224, 2010.

[15] Kun-Ming Yu, Chang Wu Yu and She-Feng Yan, "An Adhoc Routing Protocol with Multiple Backup Routes”, Springer, Wireless Personal Communications, Volume-57, 2011.

[16] Gabriel loan Ivascu, Samuel Pierre and Alejandro Quintero,'QoS Routing with Traffic Distribution in Mobile Adhoc Networks", Computer Communications, Vol-32, pp-306-316, 2009.

[17] Xuemei Gao, Xinming Zhang, Dong Shi, Fengfu Zou, Wenbo Zhu, “ Contention and Queue-aware Routing Protocol for Mobile Ad hoc Networks", International Conference on Wireless Communications, Networking and Mobile Computing (WiCom), pp 1628 - 1631, 2007.

[18] Vinay Rishiwal, S. Verma and S. K. Bajpai, " QoS Based Power Aware Routing in MANETs", International Journal of Computer Theory and Engineering, Vol. 1, No. 1, April 2009.

[19] Network Simulator, http://www.isi.edu/nsnam/ns 